\title{
Histochemistry of enzyme response to trauma in the neocortex and corpus callosum of developing rat brain
}

\author{
N. ROBINSON ${ }^{1}$ \\ From the Department of Anatomy, The London Hospital Medical College, London
}

SUMMARY The enzyme response to injury of the brain was well localized and limited. Some enzymes, even in 12 day old brain, increased rapidly, mainly in neocortical glial cells. In the corpus callosum enzymes were not significantly hyperactive before the light myelination stage. Some hyperactivity declined after 21 days. Oxidative processes and phosphate metabolism were most disturbed.

The effect of intracerebral penetration of a syringe needle on the metabolism of nerve and glial cells in the adult rat brain is reflected in wide variations in the response of enzyme activities to trauma, the variations persisting in the neocortex and subadjacent white matter over a period of 31 days post-injection (Robinson, 1969a).

Driving a needle through the neocortex and into the subadjacent white matter results in tissue necrosis in the line of needle penetration with diminishing effects receding from the site of necrosis. Morphological, colloidal, and chemical changes transmitted through the tissue by the needle produce disturbances of the metabolism of nerve cells, glial cells, and myelinating fibres and it is thought that the magnitude of the disturbances and the rates of recovery would be different at different stages of development. This paper describes the changes in activities of enzymes of important metabolic pathways of brain at the site of intracerebral penetration into the neocortex and corpus callosum at different stages of myelination.

\section{METHODS}

Male Sprague-Dawley rats, aged 12, 20, and 30 days old, were used. The animals were lightly anaesthetized, hair shaved off the scalp, and a sagittal incision

1 Address for reprints: Department of Anatomy, The London Hospital Medical College, London E.1. Z.A.1. made under aseptic conditions to expose the skull A burr-hole was drilled in the skull to expose the dura mater at area 4 of Krieg (1946) and a sterilized Luer $27 \mathrm{G}$ needle introduced to a predetermineof depth to penetrate the neocortex and corpus callo 0 sum. The needle was withdrawn and 300,000 i.u. benzathine penicillin was administered intraperio̊ toneally before suturing the incision; animals killee one day after injection were not sutured.

The animals were killed at intervals of $1,2,4,8$, 14,21 , and 31 days after operation. The brains were rapidly dissected, instantly frozen and fresh frozen sections $10 \mu \mathrm{m}$ thick were cut on a cryostat. The enzymes examined by techniques previously described (Robinson, 1969b) were as follows: $\mathrm{NADH}_{2}$ diaphorase, glucose 6-phosphate (G6-PDH), lactate (LDH), succinate (SDH) and $\alpha$-glycerophosphate $\left(\alpha\right.$-GPDH) dehydrogenases, acid phosphatase, $\mathbf{M g}^{2+}$ ATPase, 5'-nucleotidase, thiamine pyrophosphatase (TPPase), monoamine oxidase (MAO), acetylcholinesterase (AChE) and non-specific esterase (NSE).

\section{RESULTS}

CLINICAL SIGNS All the animals survived the operation and showed no apparent ill-effects or abnormalities in behaviour immediately after the operation or over the period of recovery.

12 DAY OLD BRAIN Onset of myelination of corpus callosum Up to four days post-injection a line of tissue necrosis produced by penetration of the needle was seen, but at eight days the 
lesion was identified only by changes in enzyme activity.

At one day post-injection a narrow welldefined region of neocortex showed weak $\mathrm{NADH}_{2}$-diaphorase, LDH, and G6-PDH reactions in the cytoplasm of glial cells and LDH was slightly increased in neuropil; oxidative enzyme reactions in the corpus callosum were negative. Acid phosphatase showed weak activity in nerve cells, along fragmented axons, and within a few macrophages in the neocortex but no response was apparent in the corpus callosum. In the neocortex ATPase was slightly increased in swollen glial cells but the corpus callosum showed

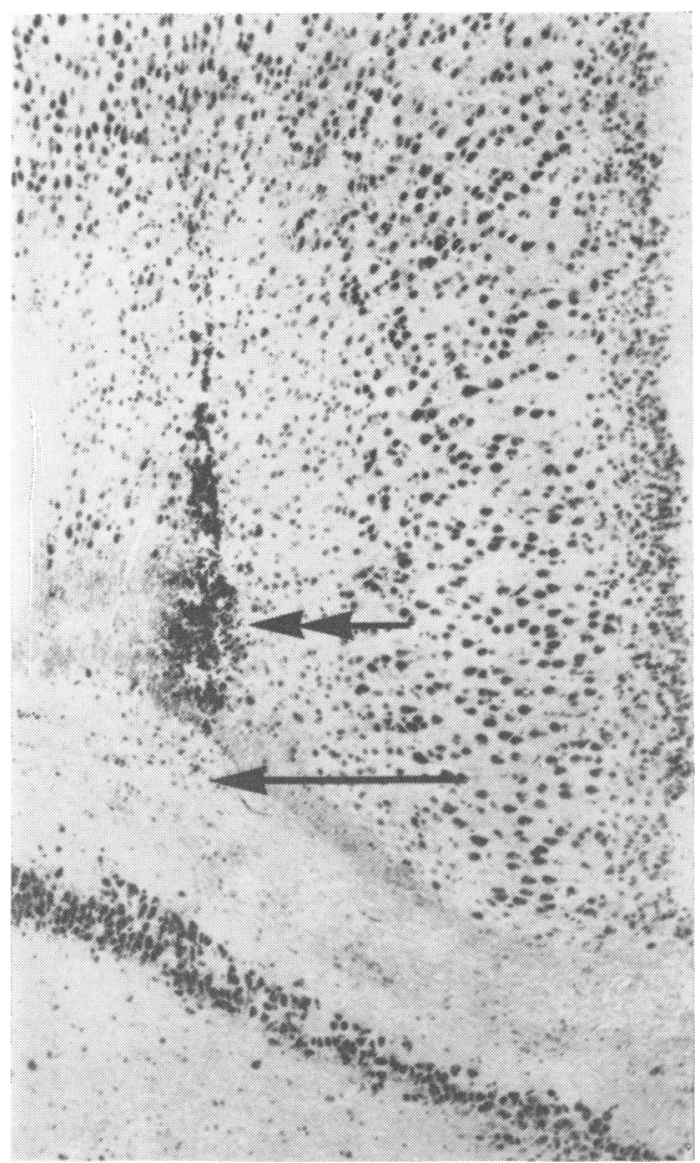

FIG. 1. Twelve day old rat; four days post-injection. Raised acid phosphatase activity in numerous cells of neocortex (double arrow) but fewer cells in corpus callosum (single arrow). $\times 50$. no response. Only a line of isolated scattered single cells showed a raised TPPase activity in the neocortex and corpus callosum. NSE activity was raised in nerve and glial cells but the localization was poor. No changes in MAO or AChE activities were detected.

By four days post-injection a raised $\mathrm{NADH}_{2}$ diaphorase activity was seen in nerve cells, in glial cells, and neuropil adjacent to tissue necrosis. Numerous glial cells in the neocortex but only scattered cells in the corpus callosum exhibited raised acid phosphatase activity (Fig. 1). A poorly localized moderately increased ATPase activity was seen in scattered glial cells

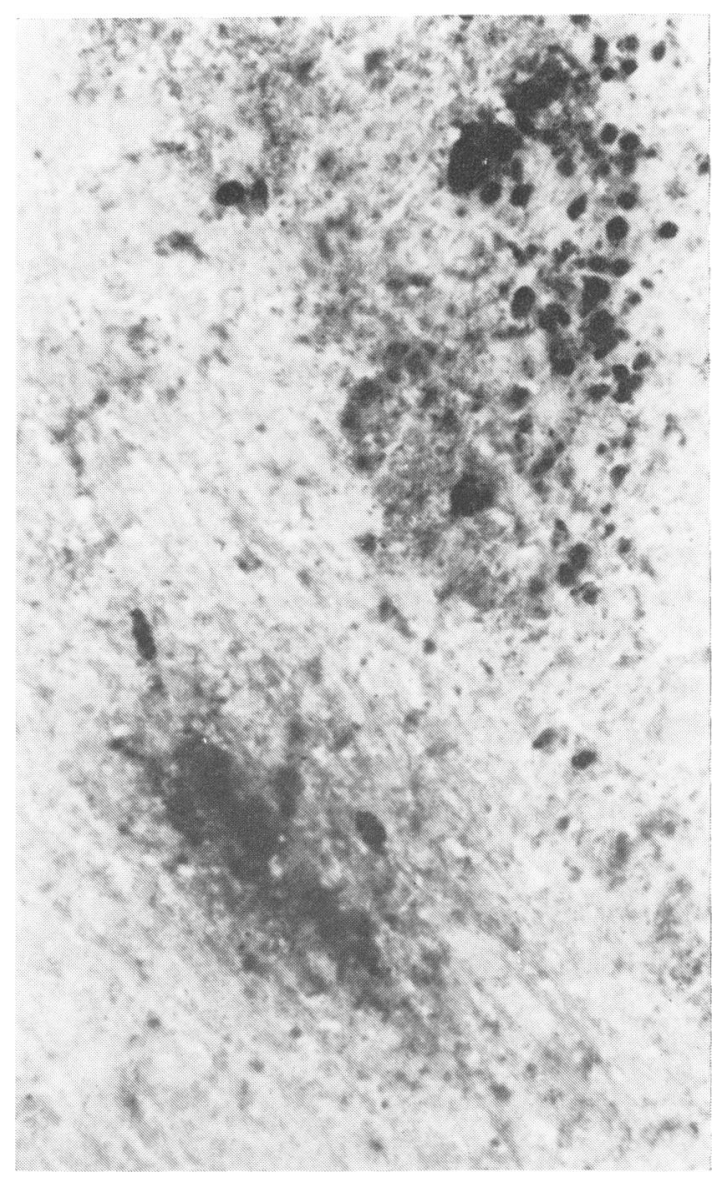

FIG. 2. Twelve day old rat; four days post-injection. Well-defined raised 5'-nucleotidase activity in swollen glial cells of neocortex (above) and diffuse activity in corpus callosum (below). $\times 310$. 
in the neocortex but the cytoplasm of some nerve and glial cells receding away from the area of most activity also showed a weak response. Swollen glial cells showed prominent raised $5^{\prime}$ nucleotidase activity but nerve cells showed a similar reaction to that seen at two days. The most marked change at four days was in the corpus callosum where a poorly localized reaction, but with recognizable glial cells, showed increases in 5'-nucleotidase; some fibres also showed the enzyme reaction, albeit weaker, not normally seen (Fig. 2). TTPase in nerve cells along the lesion had increased slightly but other structures showed no distinct response (Fig. 3).

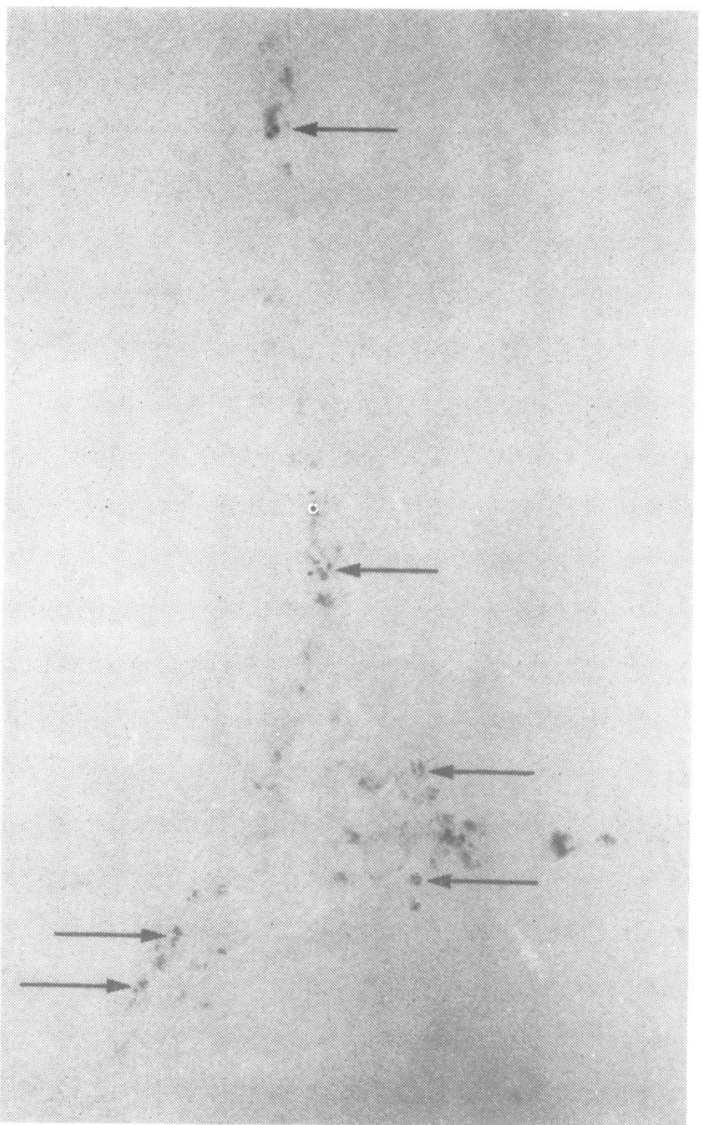

FIG. 3. Twelve day old rat; four days post-injection. Sparse nerve cells (arrows) with slightly raised thiamine pyrophosphatase activity. $\times 125$.
Other enzymes showed no marked changes except a slight increase in MAO activity in some nerve cells in the neocortex (Fig. 4).

By eight days, only raised enzyme activities enabled the lesion to be identified, since foci of damaged tissue were seldom seen. In the neocortex a more intense ATPase reaction was seen in glial cells and in some axons in the neocortex and also more numerous astrocytes with raised 5 '-nucleotidase and TTPase activities. No significant changes in the corpus callosum were observed.

20 DAY OLD BRAIN Light myelination of corpus

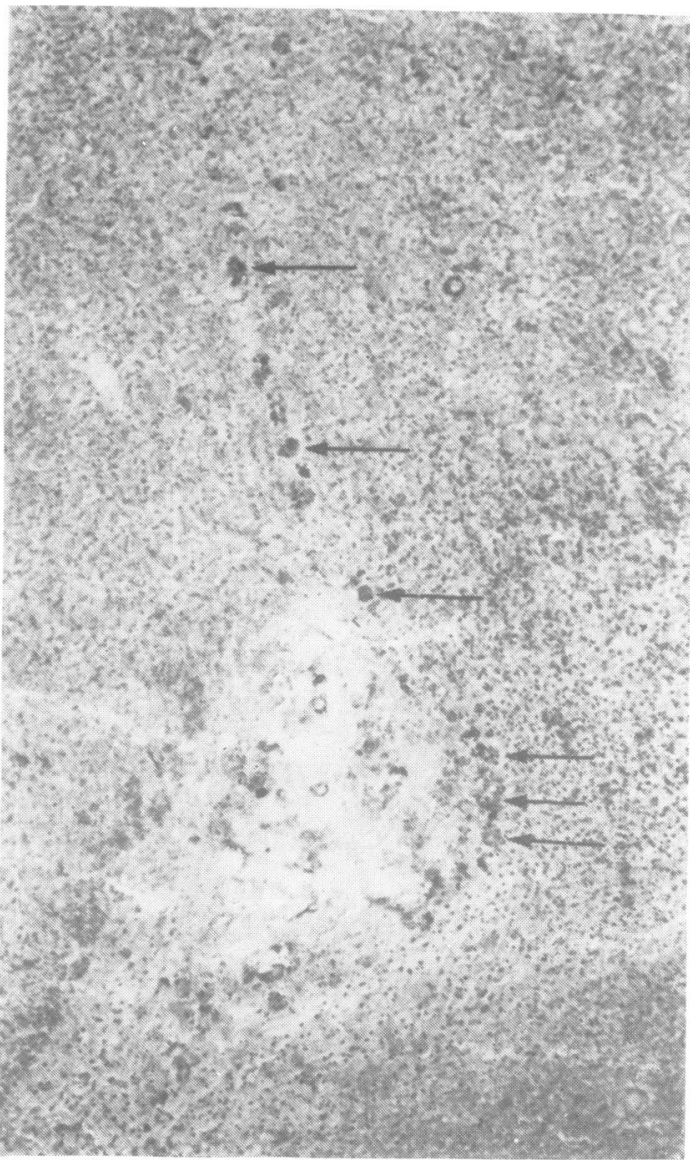

FIG. 4. Twelve day old rat; four days post-injection. Slightly raised $M A O$ activity in a few nerve cells (arrows) similar to Fig. 3. $\times 125$. 
callosum The response of oxidative enzymes to disturbance was more marked than in 12 day old animals. The reactions of these enzymes in the neocortex one day post-injection were generally still weak but the localization was more distinct; more glial cells showed increased oxidative enzyme activity, particularly $\mathrm{NADH}_{2}$-diaphorase (Fig. 5), but the neuropil remained unchanged. In the corpus callosum swollen glial cells with mainly $\mathrm{NADH}_{2}$-diaphorase, $\mathrm{LDH}$, and $\alpha$ GPDH activities were seen for the first time at one day post-injection; some oligodendrocytes also showed a reaction but there was no response in nerve fibres. The reactions of phosphatases in

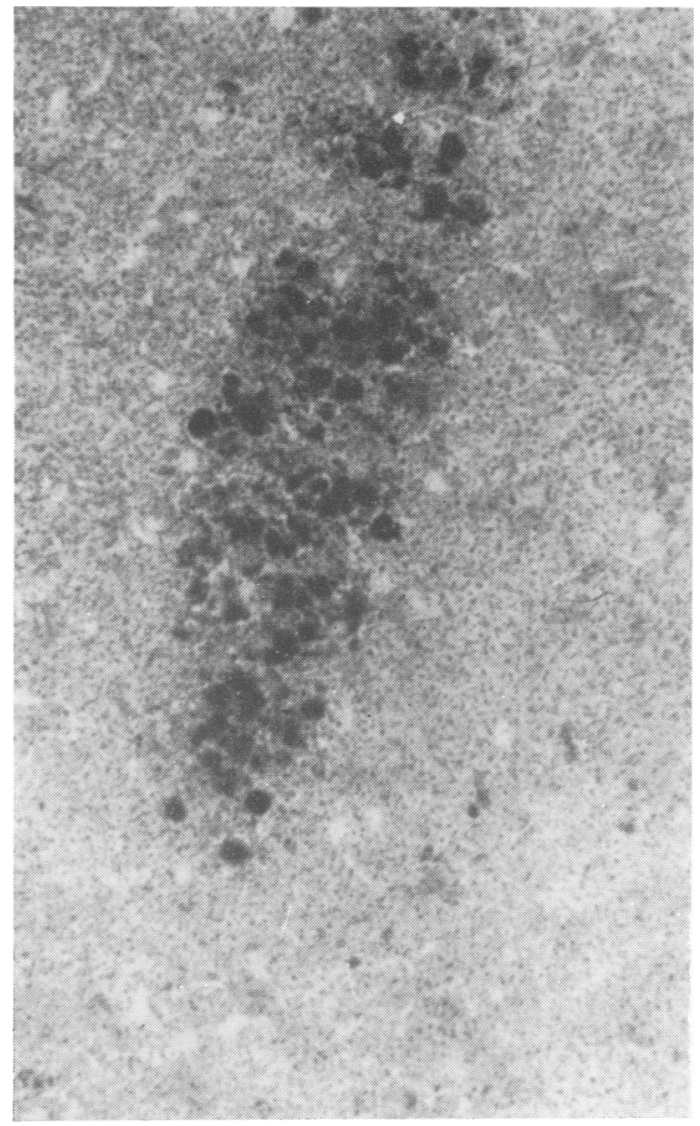

FIG. 5. Twenty day old rat; one day post-injection. Strong $\mathrm{NADH}_{2}$-diaphorase activity mainly confined to glial cells in the neocortex. $\times 310$ unaffected neocortex were generally more intense but the activities in response to injury were only slightly raised, comparable with the 12 day old brain. In the corpus callosum a marked acid phosphatase response in swollen glial cells and nerve fibres was seen and 5 '-nucleotidase showed a prominent glial cell reaction at one day postinjection. Other enzymes of phosphate metabolism showed little change except within a few scattered cells. A raised NSE activity in affected nerve cells was more precisely localized than in 12 day old brain and several prominent glial cells exhibited raised activities in the corpus callosum. Other enzymes, particularly NSE,

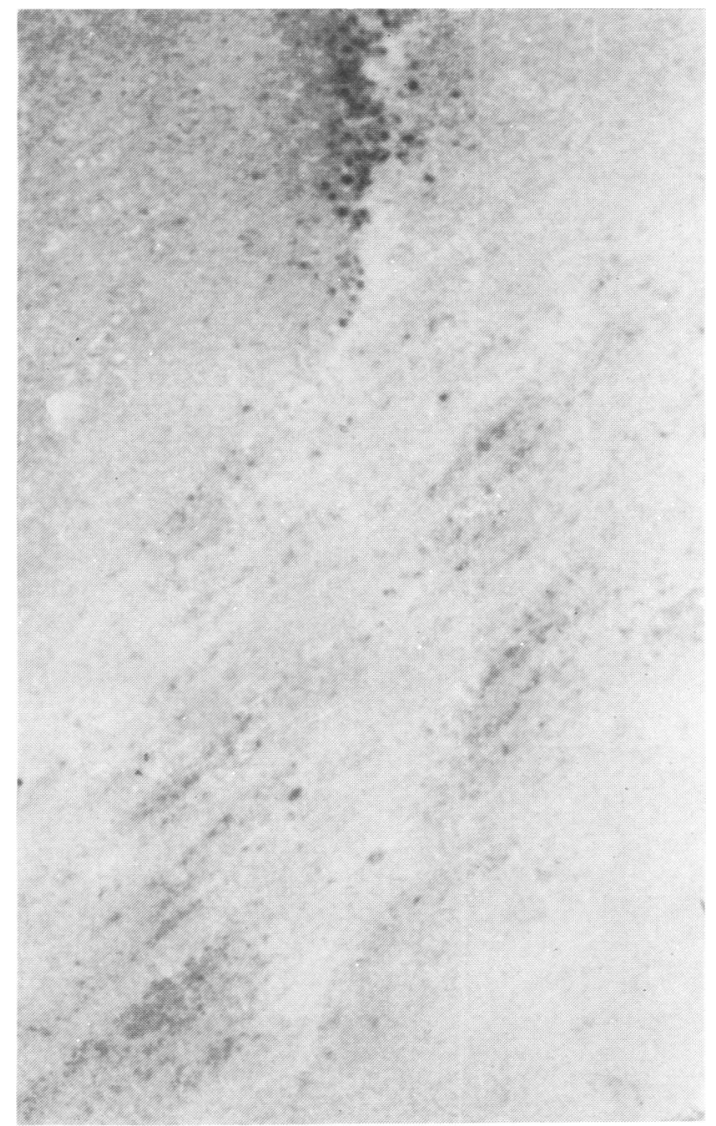

FIG. 6. Twenty day old rat; one day post-injection. Moderately raised $L D H$ activity in neocortex and slightly raised activity in oligodendrocytes and nerve fibres of corpus callosum. $\times 125$. 
MAO, and AChE, did not show any significant increases in activity up to 14 days post-injection. At 21 days post-injection the most significant change was a decrease in number of cells with raised enzyme activities.

30 DAY OLD BRAIN Substantial myelination of corpus callosum More rapid increases in enzyme activities were observed in both neocortex and corpus callosum in response to injury at this age than in younger animals; the lesion also appeared more extensive than before.

At one day post-injection $\mathrm{NADH}_{2}$-diaphorase activity was stronger in many more swollen glial

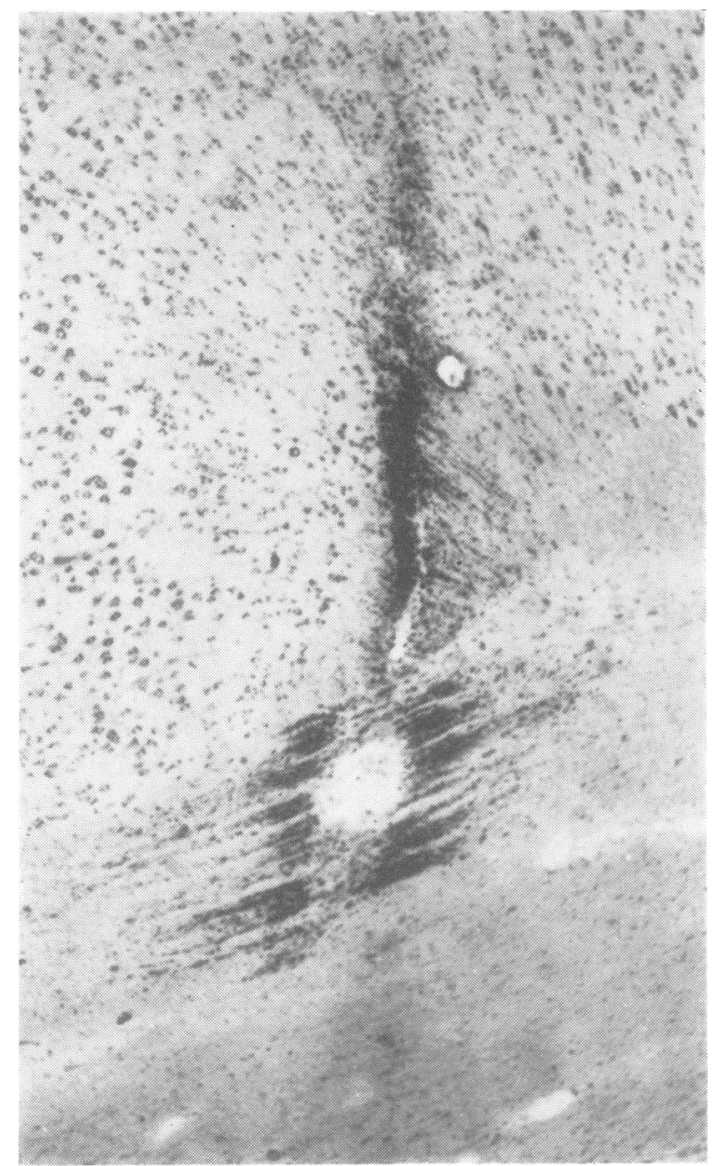

FIG. 7. Thirty day old rat; one day post-injection. Abnormally intense acid phosphatase activity in glial cells of neocortex and glial cells and nerve fibres in corpus callosum. $\times 50$. cells of the neocortex than in younger animals and the strong reaction in neuropil frequently masked the response in nerve cells. The reaction of $\mathrm{LDH}$ to injury was less intense than $\mathrm{NADH}_{2}-$ diaphorase in the neocortex; in the corpus callosum a well-localized slightly raised LDH activity was seen in lines of oligodendrocytes and nerve fibres (Fig. 6). In the neocortex and corpus callosum acid phosphatase activity was abnormally strong within glial cells and many fibres (Fig. 7). In the corpus callosum raised acid phosphatase activity was seen some length along

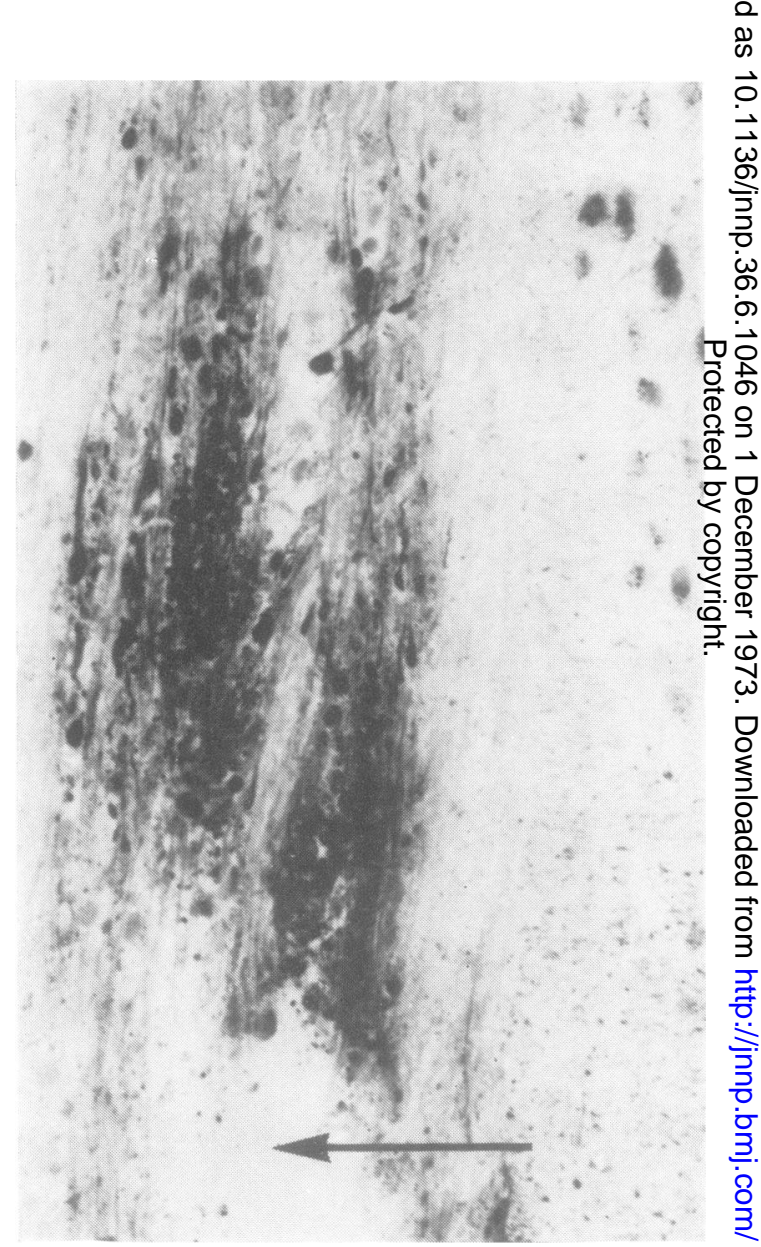

FIG. 8. Thirty day old rat; one day post-injection. Intense acid phosphatase activity (normally absent) in swollen glia and nerve fibres adjacent to injury in corpus callosum (left hand side). Poorly localized activity in nerve cells at top right hand side. Arrow denotes needle penetration. $\times 310$. 
nerve fibres receding from the lesion accompanied by hyperactive glial cells (Fig. 8). ATPase and 5'-nucleotidase reactions were similar to those in the 20 day old rats, while TPPase, NSE, MAO, and AChE showed no distinguishing reactions. At four days post-injection the distribution and raised intensity of G6-PDH in the neocortex was similar to other oxidative enzymes but in the corpus callosum a raised G6-PDH activity was mainly within glial cells close to injury (Fig. 9) and not along nerve fibres as for LDH in Fig. 6 and acid phosphatase in Fig. 7. Raised ATPase activity was seen in few glial cells and was negligible in neuropil and in nerve fibres in contrast with acid phosphatase.

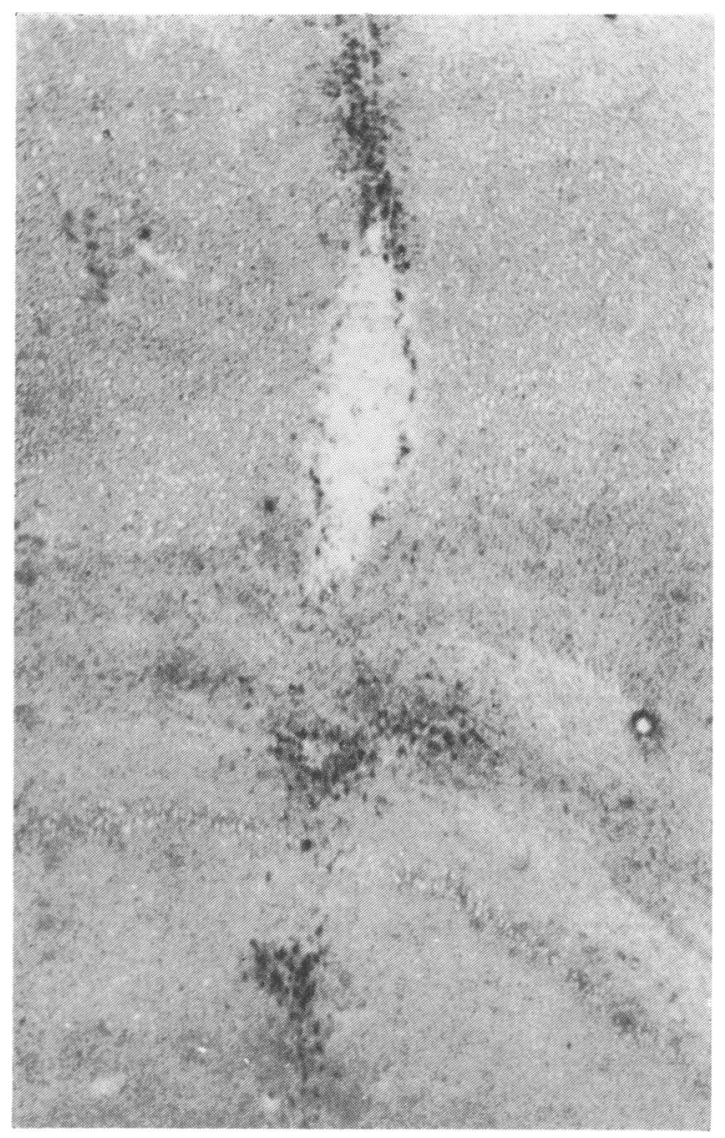

FIG. 9. Thirty day old rat; four days post-injection. Raised glucose 6-phosphate dehydrogenase activity in glial cells of neocortex and corpus callosum with no response in nerve fibres (compare LDH in Fig. 6). $\times 50$.
At subsequent times the corpus callosum enzymes showed further increases, particularly $\mathrm{NADH}_{2}$-diaphorase, LDH, and acid phosphatase; ATPase and acid phosphatase showed more distinct raised activities along nerve fibres, some appearing enlarged with strong reactions which were normally absent. TTPase showed an unusual well-localized raised activity in a few scattered swollen glial cells at 21 days postinjection. In the neocortex MAO was localized along the lesion as lines of beads, suggesting accumulation of the enzyme within small globules. By 21 days post-injection there were residual areas of tissue necrosis and the appearance of scarring was more in evidence in neocortex than in corpus callosum. Raised activity of phosphatases had diminished in cells but bare axons still exhibited a reaction.

\section{DISCUSSION}

In experiments on intracerebral injection, syringe needle or electrodes smaller than the Luer $27 \mathrm{G}$ are frequently employed but a substantial furrow into the cerebrum was necessary to observe recovery in young animals before morphological and enzyme changes were masked by growth of new tissue. Damage to tissue along the site of needle penetration was surprisingly limited and the response of enzyme activity in the neocortex was invariably confined to cells of the periphery of damaged tissue but in the corpus callosum the lesion was usually more extensive and the enzyme response was frequently more widely distributed.

In the corpus callosum the 20 day old rat showed the first distinct oxidative enzyme reaction along nerve fibres where lines of reactive oligodendrocytes receded from the site of injury similar to a reaction reported at the periphery of plaques of multiple sclerosis (Ibrahim and Adams, 1963; Friede and Knoller, 1964). However, the responses of the oxidative enzymes were dissimilar; LDH activity was less intense in the corpus callosum than in neocortex, G6$\mathrm{PDH}$ activity was equally intense in both regions, while $\alpha$-GPDH activity was also equally intense but within many fewer glial cells in the corpus callosum compared with neocortex. The intense acid phosphatase response in glial cells and myelinated nerve fibres also observed in other pathological states (Friede, 1962; Robinson, 
1969a) persisted up to 14 days post-injection but then diminished; the elevated ATPase and 5'nucleotidase activities also diminished in intensity and distribution, but not before 21 days. 5'nucleotidase was the only enzyme showing a distinct increase in activity in the early myelinating stage of the corpus callosum up to eight days post-injection, although raised acid phosphatase activity was apparent in scattered glial cells. After the initial appearance of this enzyme response, subsequent enzyme changes in the corpus callosum were less distinct, since the lesion was difficult to locate and was usually seen as a scarring with fewer hyperactive swollen glial cells retaining strong enzyme activity. Only in the more substantially myelinated corpus callosum was acid phosphatase seen to follow damaged fibres, in some instances as far medially as the midline.

In the developing neocortex enzyme reactions showed closer patterns of response to injury than seen at each stage of myelination in the corpus callosum, where it has been suggested that breakdown products of myelin are possible stimuli (Osterberg and Wattenberg, 1962). In older animals the number of glial cells showing enlargement and raised enzyme activity increased up to three weeks but in the 12 day old rats morphology and enzyme changes were not detectable two weeks after injection, being masked by rapid overgrowth of new tissue. During development the enzyme changes in nerve cells were generally small but those cells at the periphery of neocortical damage showed a greater increase in enzyme activity than cells more distant. Sometimes this increase was masked by a strong neuropil reaction; the nerve cell population showing abnormal reactions, however, diminished rapidly with the distance from injury. Detectable changes in MAO, NSE, AChE, and TPPase activity appeared to be more within nerve cells than glia and confined to cells in the line of needle penetration.

\section{CONCLUSION}

The extent of tissue damage due to penetration of a Luer $27 \mathrm{G}$ syringe needle was surprisingly limited and the enzyme response, which was well localized, was generally confined to the periphery of the injury. In the neocortex the response was rapid and intense for enzymes of oxidative processes and phosphate metabolism, even at the onset of myelination stage. In the corpus callosum enzyme response in the same metabolic pathways was not significant until light myelination when the response was rapid. As the corpus callosum became more myelinated, so the intensity of enzyme response to the lesion increased. The hyperactivity of some enzymes appeared to diminish 21 days after injury and evidence of some scarring was first seen; other enzymes, particularly thiamine pyrophosphatase, MAO, AChE, and non-specific esterase, showed no significant response to injury in the neocortex or corpus callosum at any stage of the developing brain.

The Friedreich's Ataxia Group and the Action for the Crippled Child Fund are thanked for financial assistance.

\section{REFERENCES}

Friede, R. L. (1962). An enzyme histochemical study of cerebral arteriosclerosis. (With some data on the pathogenesis of periarterial scars.) Acta Neuropathologica, 2, 58-72.

Friede, R. L., and Knoller, M. (1964). Quantitative enzyme profiles of plaques of multiple sclerosis. Experientia, 20, 130-132.

Ibrahim, M. Z. M., and Adams, C. W. M. (1963). The relationship between enzyme activity and neuroglia in plaques of multiple sclerosis. Journal of Neurology, Neurosurgery, and Psychiatry, 26, 101-110.

Krieg, W. J. S. (1946). Accurate placement of minute lesions in the brain of the albino rat. Quarterly Bulletin of the Northwestern University Medical School, 20, 199-208.

Osterberg, K. A., and Wattenberg, L. W. (1962). Inductive factors in gliosis. Proceedings of the Society of Biology and Medicine, 111, 452-455.

Robinson, N. (1969a). Histochemical changes in neocortex and corpus callosum after intracranial injection. Journal of Neurology, Neurosurgery, and Psychiatry, 32, 317-323.

Robinson, N. (1969b). Histochemistry of human cervical posterior root ganglion cells and a comparison with anterior horn cells. Journal of Anatomy, 104, 55-64. 